Journal of Animal and Veterinary Advances 11 (13): 2261-2267, 2012

ISSN: $1680-5593$

(C) Medwell Journals, 2012

\title{
Antimicrobial Drug Resistance Among Enterococci from Broilers and Poultry Abattoir Workers
}

\author{
${ }^{1}$ James W. Oguttu, ${ }^{2}$ Jackie Picard and ${ }^{3}$ Peter N. Thompson \\ ${ }^{1}$ Department of Agriculture and Animal Health, College of Agriculture and Environmental Science, \\ University of South Africa, Private Bag X11, 1710 Florida, South Africa \\ ${ }^{2}$ Department of Veterinary Tropical Diseases, ${ }^{3}$ Department of Production Animal Studies, \\ Faculty of Veterinary Science, University of Pretoria, Private Bag X04, \\ 0110 Onderstepoort, South Africa
}

\begin{abstract}
Poultry abattoir workers who carry out eviscerate do pick up resistance from broiler enteric organisms. To test this hypothesis, the prevalence of and the association of antimicrobial drug resistance between enterococci from broilers and workers who carry out evisceration, washing and packing of broiler intestines were investigated. Broiler caecae $(n=240)$ from 6 farms were collected after slaughter. Caecal content as well as faeces from 29 abattoir workers and 28 human controls were selectively cultured for E. faecium and E. faecalis. The micro-dilution broth method was used to determine MICs for selected antimicrobials. Broilers carried higher levels of resistance for certain antimicrobials compared to the two human groups. Percent resistance and $\mathrm{MIC}_{90}$ for enrofloxacin and bacitracin and $\mathrm{MIC}_{50}$ for doxycycline showed that abattoir workers carried higher levels of resistance compared to the control group for antimicrobials used in poultry production. Resistance levels in the isolates from broilers and abattoir workers exhibited an association for certain drugs. Overall, the level of resistance in the two human populations did not exhibit a significant difference ( $>0.05)$. Usage of antimicrobials as feed additives fuels resistance among broiler isolates. Abattoir workers are more likely to carry higher levels of resistance than the general public. However, this study did not demonstrate that carrying out evisceration, washing and packing of intestines of broilers fed antimicrobial feed additives significantly influences the level of resistance in abattoir workers.
\end{abstract}

Key words: Abattoir workers, broilers, enterococci, antimicrobial resistance, level of resistance

\section{INTRODUCTION}

Antimicrobial resistance is commonly observed among commensal bacteria from intensively-reared food animals (De Oliveira et al., 2004; Kalter et al., 2010) and there is a possibility that this resistance could be passed on to animal handlers (Neely and Holder, 1999). Furthermore, there is a potential for antimicrobial usage in animals to induce cross-resistance to antimicrobials used in human medicine (Anderson et al., 2003; Chen et al., 2002; Wegener et al., 1999).

In sub-Saharan Africa antimicrobial resistance is increasingly recognised in pathogens that cause infections in health-care settings, rendering the first-line and cheaper antimicrobials ineffective (Byarugaba, 2004). This is problematic given that there is a high incidence of infectious diseases in developing countries caused by a multitude of microorganisms that include bacteria (Katende-Kyenda et al., 2006) and that a large proportion of the humans in sub-Saharan Africa live below the bread-line and many are affected with the Human Immunodeficiency Virus (HIV) hence susceptible to life-threatening opportunistic bacterial infections. Therefore, infections due to resistant bacteria is likely to be associated with increased mortalities and prolonged hospital stays due to delays in administration of the correct antimicrobials (Byarugaba, 2004; Mayrhofer et al., 2004; Nugent and Okeke, 2010). Including antimicrobials as feed additives in the diet of food animals is one of the drivers of resistanc (Bogaard van den et al., 2002; Kasimoglu-Dogru et al., 2010). As a result, many developed nations including Canada, Australia and countries in Europe have banned the practice.

Escherichia coli and the thermophilic enterococci that are not only ubiquitous but have the ability to exchange resistant plasmids with related bacteria, e.g., human pathogens are used in surveillance programs for antimicrobia 1 resistance (Kasimoglu-Dogru et al., 2010;

Corresponding Auhtor: James W. Oguttu, School of Veterinary and Biomedical Sciences, Faculty of Medicine, Health and Molecular Sciences, James Cook University, 4811 Townsville, QLD, Australia 
Aarestrup et al., 2001; Nel, 2002; WHO, 2000). While in the developed countries, there is a lot of information on resistance among isolates from food animals in developing nations including South Africa, the response has been very slow due to lack of resources and the need to attend to other pressing needs.

As a result, there is paucity of data on the subject more so on people who closely work with animals and are constantly in touch with potentially resistance enteric microbes.

To assess whether working in a poultry abattoir could influence the level of resistance among enteric organisms of abattoir workers, researchers investigated the prevalence of and the association between antimicrobial drug resistances among enterococci from broilers fed antimicrobial feed additives and abattoir workers who carry out the evisceration washing and packing of intestines from broilers after slaughter.

\section{MATERIALS AND METHODS}

Specimen collection: Collection of specimens from broilers and humans started in June, 2005 and was finalised in June, 2006. Broiler farms ( $n=6$ ) from different grow-out-cycles were sampled at one high-throughput poultry abattoir in Gauteng province, South Africa. Caecae were harvested from carcasses passed as fit for human consumption. Caecae $(n=240)$ were collected every 3-5 min after slaughter by excising them off carcasses using sterile scissors and forceps. Each caeca was tied off at the open end and placed separately in a labelled, sterile plastic bag then placed in a cooler box with ice packs.

Abattoir workers who eviscerate wash and pack intestines and had not been on antimicrobial therapy during the 3 months prior to sampling were asked to provide a faecal sample. Total 29 of 44 people completed informed consent forms and participated in the study. The control group (students and workers at the Faculty of Veterinary Science, University of Pretoria) had not received antimicrobial treatment and had not been in direct contact with poultry fed with antimicrobial feed additives 3 months prior to sampling. About 28 people completed informed consent forms and participated in the study.

The faecal sample was collected by the subjects themselves by scooping off the first or last faeces from the anal area using sterile plastic spoons supplied to them.

Samples were placed in sterile bottles which were dropped off as the workers reported for work. Samples were conveyed on frozen ice packs to the bacteriology laboratory of the Department of Veterinary Tropical Diseases, Faculty of Veterinary Science and processed the same day.

Culture and identification of Enterococcus sp.: Approximately $0.5 \mathrm{~g}$ caecal content or faeces from each sample was plated on Kanamycin Aesculin Azide agar (KAA) plates (Oxoid, UK), a selective medium for Enterococcus sp. and incubated at $45^{\circ} \mathrm{C}$ for $18-24 \mathrm{~h}$. One presumptive colony of Enterococcus was then plated onto Columbia agar (Sigma-Aldrich, USA) containing 7\% citrated horse blood and incubated at $37^{\circ} \mathrm{C}$ for $18-24 \mathrm{~h}$. Pin-point white colonies with a zone of beta-haemolysis, containing gram-positive, coccoid, catalase-negative, non-motile organisms belonging to group $\mathrm{D}$ were identified as enterococci.

Enterococcus faecalis and E. faecium were differentiated using a panel of sugar fermentation tests (Manero and Blanch, 1999).

Antimicrobial susceptibility testing: The Clinical Laboratory Standards Institute (CLSI) micro-broth dilution method (NCCLS, 1994) was used to determine the Minimum Inhibitory Concentration (MIC). The isolates were classified as either susceptible or resistant depending on whether the $\mathrm{MIC}$ was higher or lower than microbiological cut-off recommended by CLSI or the surveillance programmes of some European Union countries (NCCLS, 1994; DANMAP, 2009). The following cut-off points were used: vancomycin $16 \mathrm{mg} \mathrm{L}^{-1}$, ampicillin $8 \mathrm{mg} \mathrm{L}^{-1}$, ceftriaxone $1 \mathrm{mg} \mathrm{L}^{-1}$, doxycycline $8 \mathrm{mg} \mathrm{L}^{-1}$, bacitracin $25 \mathrm{IU} \mathrm{mL}^{-1}$, erythromycin $4 \mathrm{mg} \mathrm{L}^{-1}$, enrofloxacin $0.25 \mathrm{mg} \mathrm{L}^{-1}$, fosfomycin $16 \mathrm{mg} \mathrm{L}^{-1}$, sulphamethoxazole $256 \mathrm{mg} \mathrm{L}^{-1}$ and trimethoprim $8 \mathrm{mg} \mathrm{L}^{-1}$.

Data analysis: Stata 8.2 statistical software (StataCorp, College Station, TX, USA) was used for analysis. Fisher's exact test was used to compare percentages of antimicrobial drug resistance in the three populations. A $\mathrm{p}<0.05$ was considered statistically significant.

The $\mathrm{MIC}_{50}$ and $\mathrm{MIC}_{90}$ (MIC at which 50 and $90 \%$ of the strains were inhibited by the respective antimicrobial) were determined to assess shifts in antimicrobial susceptibility.

\section{RESULTS AND DISCUSSION}

Prevalence of resistance: The two species (E. faecalis and $E$. faecium) were combined and hence referred to as enterococci. High resistance was observed among broilers (Table 1) to classes of antimicrobials extensively used in broilers in South Africa, i.e., doxycycline (96.6\%), 
J. Anim. Vet. Adv., 11 (13): 2261-2267, 2012

Table 1: Percentage of Enterococcus isolates resistant with exact binomial $95 \%$ confidence interval, amongst broilers, poultry abattoir workers and human controls

\begin{tabular}{|c|c|c|c|}
\hline \multirow[b]{2}{*}{ Antimicrobial drug } & \multicolumn{3}{|l|}{ Human groups } \\
\hline & Broilers & Abattoir workers & Controls \\
\hline Vancomycin & $2.6^{a}(0.5-7.30)$ & $8.7^{\mathrm{a}}(1.1-28.0)$ & $0.0^{\mathrm{a}}(0.0-20.6)$ \\
\hline Virginiamycin & $0.0^{\mathrm{a}}(0.0-3.10)$ & $0.0^{\mathrm{a}}(0.0-77.6)$ & $0.0^{\mathrm{a}}(0.0-25.9)$ \\
\hline Bacitracin & $47.4^{\mathrm{a}}(38.1-56.9)$ & $8.7^{b}(1.1-28.0)$ & $0.0^{b}(0.0-20.6)$ \\
\hline Doxycycline & $96.6^{\mathrm{a}}(91.4-99.1)$ & $60.9^{b}(38.5-80.3)$ & $46.2^{\mathrm{b}}(19.2-74.9)$ \\
\hline Trimethoprim & $3.4^{a}(0.9-8.60)$ & $30.4^{b}(13.2-52.9)$ & $7.7^{\mathrm{ab}}(0.2-36)$ \\
\hline Sulphamethoxazole & $88.8^{a}(81.6-93.9)$ & $73.9^{a}(51.6-89.8)$ & $76.9^{\mathrm{a}}(46.2-95)$ \\
\hline Ampicillin & $10.3^{\mathrm{a}}(5.5-17.4)$ & $0.0^{\mathrm{a}}(0.0-12.2)$ & $7.7^{a}(0.2-36)$ \\
\hline Enrofloxacin & $86.2^{\mathrm{a}}(78.6-91.9)$ & $56.5^{b}(34.5-76.8)$ & $46.2^{\mathrm{b}}(19.2-74.9)$ \\
\hline Erythromy cin & $100.0^{\mathrm{a}}(97.5-100)$ & $78.3^{\mathrm{b}}(56.3-92.5)$ & $92.3^{\mathrm{ab}}(64-99.8)$ \\
\hline Fosfomy cin & $98.3^{\mathrm{a}}(93.9-99.8)$ & $91.3^{\mathrm{a}}(72-98.9)$ & $100.0^{\mathrm{a}}(79.4-100)$ \\
\hline Ceftriaxone & $87.9^{a}(80.6-93.2)$ & $60.9^{b}(38.5-80.3)$ & $84.6^{\mathrm{ab}}(54.6-98.1)$ \\
\hline
\end{tabular}

${ }^{\mathrm{a}-c}$ Values within a row with no superscripts in common differ significantly $(\mathrm{p}<0.05)$

Table 2: Comparison of the $\mathrm{MIC}_{50}$ and $\mathrm{MIC}_{90}$ for Enterococcus isolates from broilers, poultry abattoir workers and human controls

\begin{tabular}{|c|c|c|c|c|c|c|c|}
\hline \multirow[b]{2}{*}{ Antibiotic } & \multicolumn{2}{|c|}{ Human controls } & \multicolumn{2}{|c|}{ Abattoir workers } & \multicolumn{2}{|c|}{ Broilers } & \multirow[b]{2}{*}{ Difference association } \\
\hline & $\mathrm{MIC}_{50}$ & $\mathrm{MIC}_{90}$ & $\mathrm{M \Pi C}_{50}$ & $\mathrm{MIC}_{90}$ & $\mathrm{MIC}_{50}$ & $\mathrm{MIC}_{90}$ & \\
\hline Vancomycin & 2 & 2 & 2 & 2 & 2 & 2 & - \\
\hline Virginiamycin & 1 & 2 & 2 & 2 & 2 & 8 & $\mathrm{MIC}_{90}$ \\
\hline Doxycy cline & 1 & 64 & 16 & 64 & 32 & 64 & $\mathrm{MIC}_{50}$ (Human $\mathrm{Ab}^{*}$ broilers) \\
\hline Trimethoprim & 0.25 & 8 & 0.25 & 32 & 0.25 & 0.25 & $\mathrm{MIC}_{90}($ HumanC, HumanAb) \\
\hline Sulphamethoxazole & 2048 & 2048 & 2048 & 2048 & 2048 & 2048 & - \\
\hline Ampicillin & 0.25 & 1 & 0.25 & 0.5 & 1 & 16 & $\mathrm{MIC}_{90}$ \\
\hline Enrofloxacin & 0.25 & 1 & 0.5 & 8 & 2 & 4 & $\mathrm{MIC}_{90}$ (Human $\mathrm{Ab}$; broilers) \\
\hline Erythromy cin & 256 & 256 & 32 & 256 & 256 & 256 & $\mathrm{MIC}_{50}($ HumanC) broilers \\
\hline Fosfony cin & 128 & 128 & 128 & 128 & 128 & 128 & - \\
\hline Ceftriaxone & 8 & 32 & 8 & 128 & 32 & 128 & $\mathrm{MIC}_{90}$ (HumanAb; broilers) \\
\hline Bacitracin & 3.13 & 12.5 & 3.13 & 50 & 25 & 100 & $\mathrm{MIC}_{50}$ \\
\hline
\end{tabular}

Human $\mathrm{Ab}^{*}=$ Human Abattoir workers; HumanC\# = Human Control group

sulphamethoxazole $\quad(88.8 \%)$, bacitracin $\quad(47.4 \%)$, ampicillin (10.3\%), erythromycin $(86.2 \%)$, enrofloxacin $(86.2 \%)$, fosfomycin $(98.3 \%)$ and ceftriaxone $(87.9 \%)$. Resistance to trimethoprim was lower in all the three populations compared to that of sulphamethoxazole (Table 1). Vancomycin a drug rarely used in the treatment of human infections in South Africa and its analogue avoparcin last used in poultry in South Africa 7 years prior to this study exhibited low resistance levels (Table 1) in all host populations (broilers: $2.6 \%$; abattoir workers: $8.7 \%$ and control: $0 \%$ ).

Abattoir workers carried higher levels of resistance to doxycycline (60.9\%), trimethoprim (30.4\%), bacitracin (8.7\%) and enrofloxacin (56.5\%) compared to the control $(46.2,7.7,0$ and $46.2 \%$, respectively) (Table 1 ). Contrary to what was anticipated, the control group carried higher resistance (Table 1) for sulphamexazole $(76.9 \%)$, ampicillin $(7.7 \%)$, erythromycin $(92.3 \%)$ and ceftriaxone $(84.6 \%)$ compared to abattoir workers who carried resistance of $73.9,0,78.3$ and $84.6 \%$, respectively. However, resistance (Table 1) among isolates from abattoir workers and the control group showed no significant difference $(\mathrm{p}>0.05)$. The $\mathrm{MC}_{90}$ for isolates from abattoir workers (Table 2 and Fig. 1) for bacitracin and enrofloxacin tended towards those observed among poultry isolates.
The $\mathrm{MLC}_{50}$ for doxycycline, virginiamycin and enrofloxacin (Table 2 and Fig. 2) among isolates from abattoir workers also, tended to equal that observed in broilers.

Classes of antimicrobials that were studied included potentiated sulphonamides, ampicillin, tetracyclines, enrofloxacin, ceftriaxone, erythromycin and vancomycin. The last three are available for use only in humans in South Africa. Ceftriaxone, a 3rd generation cephalosporin can develop cross resistance when isolates are exposed to other $\beta$-lactams. Tetracycline is available over the counter for use in animals as feed additives. In human medicine all antimicrobials are scheduled drugs, available only with prescription. Furthermore where prescriptions of $\beta$-lactams are required in human medicine, cephalosprins are likely to be prescribed as compared to tetracyclines. Macrolides, tetracyclines and sulphonamides were represented by erythromycin, doxycycline and sulphamethoxazole, respectively. The more stable ampicillin represented the $\beta$-lactams. Virginiamycin was not used in the flocks studied but is registered as an Antimicrobial Performance Enhancer (AMPE) in South Africa. However because of its potential to cause cross resistance to synercid, macrolides and lincosamides, it was withdrawn in the European 


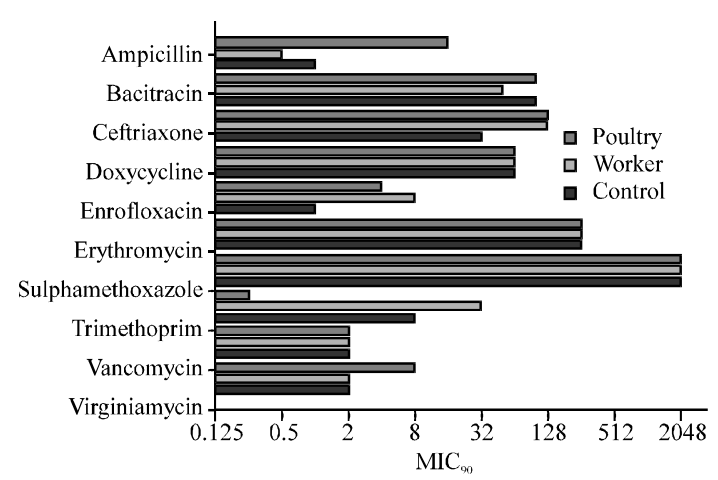

Fig. 1: $\mathrm{MIC}_{90}$ of Enterococcus isolates from broilers, abattoir workers and human controls

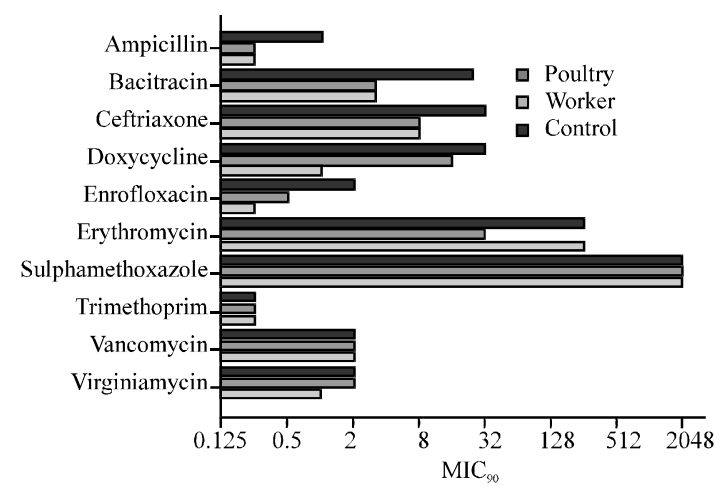

Fig. 2: $\mathrm{MIC}_{50}$ of Enterococcus isolates from broilers, abattoir workers and human controls

Union (Chen et al., 2002). Bacitracin was the only AMPE used in the flocks studied. Broilers are fed on AMPE almost their entire life and also have high use of antibiotics on veterinary prescription. This is known to select for resistance (Bogaard van den et al., 2002) and hence explains the high resistance to both therapeutic antimicrobials and AMPE observed among broilers isolates in this study. Furthermore, since the birds studied were not exposed to virginiamycin, higher resistance to bacitracin compared to virginiamycin could be attributed to the higher selection pressure exerted as a result of exposure to bacitracin.

High erythromycin resistance $(100 \%)$ observed in broilers is consistent with what others have reported (Bogaard van den et al., 2002; Kasimoglu-Dogru et al., 2010). This could be due to cross resistance due to its derivative tylosin that is routinely used to treat Mycoplasma sp., infections in poultry. Low level vancomycin resistance $(2.6 \%)$ was anticipated even though its analogue, avoparcin had been withdrawn. In South Africa (SANVAD, 2007), Denmark (Kasimoglu-Dogru et al., 2010; Aarestrup et al., 2001; Bonten et al., 2001) and Finland (Neely and Holder, 1999) persistent antimicrobial drug resistance to certain drugs was observed several years after they were withdrawn. Though, Vancomycin Resistant Enterococci (VRE) no longer have a competitive advantage they have not been totally eliminated following the withdrawal of the drug (Neely and Holder, 1999).

A higher $\mathrm{MLC}_{90}$ for virginiamycin in broilers compared to the humans could be due to resistance elements persisting on the farms or to the presence of E. faecalis among poultry isolates known to be intrinsically resistant to streptogramin like virginiamycin (McDonald et al., 2001). Lower resistance to trimethoprim compared to sulphamethoxazole when flocks were exposed to potentiated sulphonamides could be attributed either to resistance development among enterococci to the trimethoprim taking place at a slower rate or to the selection pressure for sulphonamides being higher since, sulphonamides are added to poultry feed to control coccidiosis. Consistent with reports that people working with animals tend to carry higher levels of resistance compared to the general public (Kalter et al., 2010; Bogaard van den et al., 2002), abattoir workers carried higher resistance to most antimicrobials compared to the controls.

However, a comparative analysis did not show a significant difference between the level of resistance among abattoir workers and the control group. The possible explanations for this include that eviscerating, washing and packing of intestines of broilers fed antimicrobial feed additives does not influence the level of resistance among the abattoir workers it has been reported that large numbers of immunocompromised patients and their frequent exposure to antibiotics against which enterococci are intrinsically resistant (Bogaard van den et al., 2002; Kasimoglu-Dogru et al., 2010) contribute to heightened resistance in the general public. Studies have shown that poultry $E$. coli is able to colonise humans more easily compared to enterococci (Kalter et al., 2010; Bogaard van den et al., 2002). This could also explain the failure to observe a significant difference in the level of resistance among abattoir workers and the controls.

Reduced susceptibility to bacitracin among abattoir workers compared to the controls and the fact that the $\mathrm{MIC}_{90}$ for bacitracin and the $\mathrm{MLC}_{50}$ for virginiamycin of isolates from abattoir workers were closer to those of broilers, suggests the existence of an association. Failure to observe resistance to virginiamycin in the human population in this study is consistent with results of a study done in North America that showed little resistance to quinupristin-dalfopristin among enterococci isolated from people despite decades of virginiamycin use in farm animals (McDonald et al., 2001). 
Since, cephalosporins have very weak activity against enterococci (Bogaard van den et al., 2002; Murray, 1990), high resistance to this antimicrobial was thus, anticipated in all three populations. In poultry and the control group, the predominance of $E$. faecium which is more resistant than $E$. faecalis and exhibits multi-resistance (Bogaard van den et al., 2002), accounts for the higher resistance to ceftriaxone in the two populations. However, on consideration of the $\mathrm{MIC}_{90}$ for this drug, an association of resistance between broilers and workers is evident. Penicillins constitute about $40 \%$ of the antimicrobials prescribed for treatment of respiratory tract infections in humans in South Africa (Katende-Kyenda et al., 2006). This notwithstanding, the levels of resistance to amoxicillin were very low in the study. The slightly higher resistance among the control group compared to the abattoir workers could be attributed to the former carrying more $E$. faecium known to be resistant to penicillins.

Trimethoprim/sulphonamide combinations make up $23 \%$ of antimicrobial prescriptions for the treatment of human respiratory tract infections in South Africa (Katende-Kyenda et al., 2006). In view of this, it was expected that resistance to both trimethoprim and sulphamethoxazole would be fairly similar. On the contrary resistance to trimethoprim was fairly low compared to sulphamethoxazole. As mentioned for broiler isolates, this suggests that resistance to the two parts of potentiated sulphonamides develops at different rates.

Given that doxycycline can only be obtained by prescription and that it is rarely used in human medicine in South Africa fairly high levels of resistance observed in the two human groups were not anticipated. Tetracycline resistance transfer takes place through several mechanisms, e.g., plasmid transfer. Therefore, workers can pick up resistance elements from broiler isolates. This explains the association between resistance in broilers and abattoir workers as demonstrated by the $\mathrm{MIC}_{50}$ of their isolates. Hence, usage of antimicrobials as feed additives by the broiler industry has potential to contribute to resistance observed among humans. Resistance transfer among fluoroquinolones unlike for tetracycline is mediated by chromosomal mutations (Wilcks et al., 2005). Hence, an association of resistance between broilers and abattoir workers as demonstrated by both the $\mathrm{MC}_{50}$ and $\mathrm{MIC}_{90}$ for enrofloxacin was not anticipated.

In humans, fosfomycin is used as a 3rd choice empirical therapy (Knottnerrus et al., 2008) for the treatment of acute, uncomplicated urinary tract infections. Where fosfomycin has been used for many years, resistance is still low (Knottnerrus et al., 2008). The high levels of resistance in this study could be due to a technical error. Fosfomycin activity is dependent upon a glucose-6-phosphate-induced transport system which enhances penetration of the drug into bacterial cells. A loss of this transport abolishes the action of fosfomycin (Yeo, 1988). It is thus recommended that testing of fosfomycin be done in the presence of glucose-6phosphate $\left(25 \mathrm{mg} \mathrm{L}^{-1}\right.$ ) (Greenwood et al., 1992) to facilitate absorption of the drug by the bacteria. A high resistance to fosfomycin is postulated to be possible if all the isolates are mutants lacking this transport system (Yeo, 1988). Fosfomycin is not known to have crossresistance with other antibiotics because it bears no chemical relationship to any of them. Hence, the high resistance can not be attributed to cross-resistance (Knottnerrus et al., 2008; Yeo, 1988).

Although, abattoir workers like people who work with animals on antimicrobials generally carried higher resistance levels than the control (Bogaard van den et al., 2002), the resistance level in both human populations was lower than that reported in other developing countries where antimicrobials are readily available over the counter (Okeke and Lamikanara, 2003).

\section{CONCLUSION}

Broilers exposed to antimicrobials included in their feed for therapeutic and growth enhancement purposes, carry high levels of antimicrobial drug resistance among their enteric organisms to such antimicrobials. There is an association between the resistance levels of enterococci from broilers and abattoir workers for bacitracin, ceftriaxone, enrofloxacin and doxycycline, classes of antimicrobials that are extensively used in broilers. Abattoir workers tend to carry higher levels of resistance among their enteric organisms compared to people not directly linked with the broiler industry more so, to antimicrobials frequently used as feed. However, carrying out evisceration washing and packing of intestines of broilers fed antimicrobial feed additives does not appear to significantly influence the level of resistance in abattoir workers. Vancomycin resistance genes introduced when avoparcin was extensively used in South Africa have not totally disappeared. Government and the poultry industry should work towards ensuring that avoparcin is not reintroduced for use as an AMPE in South Africa. There is a need to further investigate the role of the availability of tetracycline over the counter for use in animals in South Africa in the development of resistance against tetracycline among humans. 


\section{ACKNOWLEDGEMENT}

The researchers wish to thank Ms. Janita Greyling for her assistance in the laboratory.

\section{REFERENCES}

Aarestrup, F.M., A.M. Seyfarth, H.D. Emborg, K. Pedersen, R.S. Hendrksen and F. Bager, 2001. Effect of abolishment of the use of antimicrobial agents for growth promotion on occurrence of antimicrobial resistance in fecal enterococci from food animals in Denmark. Antimicrob. Agents Chemother., 45: 2054-2059.

Anderson, A.D., J.M. Nelson, S. Rossiter and F.J. Angulo, 2003. Public health consequences of use of antimicrobial agents in food animals in the United States. Microb. Drug Resistance, 9: 373-379.

Bogaard, van den A.E., R. Willems, N. London, J. Top and E.E. Stobberingh, 2002. Antibioitic resistance of faecal enterococci in poultry, poultry farmers and poultry slaughterers. J. Antimicrob. Chemother., 49: 497-505.

Bonten, M.J., R. Willems and R.A. Weinstein, 2001. Vancomycin-resistant enterococci: Why are they here, and where do they come from? Lancent Infect. Dis., $1: 314-325$.

Byarugaba, K.D., 2004. A view on antimicrobial resistance in developing countries and responsible risk factors. Int. J. Antimicrobial Agents, 24: 105-110.

Chen, H.Y., R.L. Hill, M. Kirk, M.W. Casewell and D. Beigton, 2002. Differential antimicrobial susceptibility between human and chicken isolates of vancomycin-resistant and sensitive Enterococcus faecium. Int. J. Antimicrob. Agents, 19: 39-46.

DANMAP, 2009. September/29/2009-last update, danish integrated antimicrobial resistance monitoring and research. http://www.danmap.org/.

De Oliveira, D.S., F.S. Flores, L.R. dos Santos and A. Brandelli, 2004. Antimicrobial resistance in Salmonella Enteritidis strains isolated from broiler carcasses, food, human and poultry-related samples. Int. J. Food Microbiol., 97: 297-305.

Greenwood, D., R. Edwards, J. Brown and P. Ridout, 1992. The comparative activity of fosfomycin trometamol against organisms isolated from infected urines. Infect., 20: S302-S304.

Kalter, D.H., H.R. Gilman, H.L. Moulton, R.A. Cullotta, L. Cabrera and B. Velapatino, 2010. Risk factors for antibiotic-resistant escherichia coli carriage in young children in peru: Community-based crosssectional prevalence study. Amer. J. Trop. Med. Hyg., 82: 879-888.
Kasimoglu-Dogru, A., Y.E. Gencay and N.D. Ayaz, 2010. Prevalence and antibiotic resistance profiles of Enterococcus species in chicken at slaughter level: Abscence of vanA and vanB genes in $\mathrm{E}$. faecalis and E. faecium. Res. Vet. Sci., 89: 153-158.

Katende-Kyenda, N.L., M.S. Lubbe, J.H. Serfontein and I. Truter, 2006. Inappropriateness of antimicrobial prescription in private primary health care settings in South Africa. South African Med. J., 96: 704-705.

Knottnerrus, B.J., S. Nys, G. ter Riet, G. Donker, S.E. Geerlings and E. Stobberingh, 2008. Fosfomycin tromethamine as second agent for the treatment of acute, uncomplicated urinary tract infections in adult female patients in The Netherlands?. J. Antimicrob. Chemother., 62: 356-359.

Manero, A. and A.R. Blanch, 1999. Identification of Enterococcus ssp. with a biochemical key. Applied Environ. Microbiol., 65: 4425-4430.

Mayrhofer, S., P. Paulsen, F.J.M. Smulders and F. Hilbert, 2004. Antimicrobial resistance profile of five major food-borne pathogens isolated from beef, pork and poultry. Int. J. Food Microbiol., 97: 23-29.

McDonald, L.C., S. Rossiter, C. Mackinson, Y.Y. Wang and S. Johnson et al., 2001. Quinupristindalfopristin-resistant entrococcus faecium on chicken and in human stool specimens. N. Engl J. Med., 345: 1155-1160.

Murray, B.E., 1990. The life and times of the enterococcus. Clin. Microbiol. Rev., 3: 46-65.

NCCLS, 1994. Performance standards for antimicrobial disk and dilution susceptibility tests for bacteria isolated from animals. Proposed Standard M31-p. National Committee for Clinical Laboratory Standards, Villanova, Pa.

Neely, A.N. and I.A. Holder, 1999. Antimicrobial resistance. Burns, 25: 17-24.

Nel, H., 2002. The establishment and standardization of a veterinary antimicrobial resistance surveillance program in South Africa. M.Sc. Thesis, University of Pretoria, Pretoria, South Africa.

Nugent, R. and I.N. Okeke, 2010. When medicines fail: Recommendations for curbing antibiotic resistance. J. Infect. Dev. Countries, 4: 355-356.

Okeke, I.N. and A. Lamikanra, 2003. Export of antimicrobial drugs by West African travelers. J. Travel Med., 10: $133-135$.

SANVAD, 2007. South African National veterinary surveillance and monitoring programme for resistance to antimicrobial drugs. SANVAD, Pretoria, South Africa. 
WHO, 2000. Drug resistance threatens to reverse medical progress. Information Office-Press Release. http://www. who.int/inf-pr-2000/en/pr2000-41.html.

Wegener, H.C., F.M. Aarestrup, L.B. Jensen, A.M. Hammerum and F. Bager, 1999. Use of antimicrobial growth promoters in food animals and Enterococcus faecium resistance to therapeutic antimicrobial drugs in Europe. Emerg. Infect. Dis., 5: 329-335.
Wilcks, A., S.R. Andersen and R.T. Licht, 2005. Characterization of transferable tetracycline resistance genes in Enterococcus faecalis isolated from raw food. FEMS Microbiol. Lett., 243: 15-19.

Yeo, M., 1988. Fosfomycin for the treatment of multidrugresistant. Singapor Med. J., 29: 91-92. 